\title{
Retrospective evaluation of trigger criteria of the rapid response system in an obstetric ward
}

\section{Yuki Hosokawa, Masayasu Arai, Rie Kato, Toshiyuki Okutomi Department of Anesthesiology, Kitasato University School of Medicine \\ Results}

\section{Background}

- Modified early obstetric warning system (MEOWS)[1]

- Is relevant to obstetric disease and adapted for normal obstetric physiological changes

- The rapid response system (RRS) at Kitasato University Hospital

- 24-h service

- Triggered by single-parameter criteria

- Designed for non-obstetric hospitalized patients

This study was aimed to assess the trigger criteria of the RRS at Kitasato University Hospital as a bedside screening tool in correlation with obstetric morbidity by comparing it with the MEOWS criteria.

\section{Methods}

$>$ We retrospectively reviewed the medical records of women admitted to MFICU at Kitasato University Hospital from January to December 2015.

Based on the recorded physiological parameters, the researcher $(\mathrm{YH})$ investigated all cases whether they met the trigger criteria of the Kitasato RRS (KRRS) and MEOWS criteria (Table 1).

Table 1. Trigger criteria of KRRS and MEOWS

\begin{tabular}{llll}
\hline & \multicolumn{2}{c}{ KRRS } & \multicolumn{2}{c}{ MEOWS } \\
\cline { 3 - 4 } $\begin{array}{lll}\text { Heart rate; } \\
\text { beats. } \text { min }^{-1}\end{array}$ & $<50$ or $>120$ & $\begin{array}{l}\text { Y0-50 } \\
\text { or } 100-120\end{array}$ & $<40$ or $>120$ \\
Systolic BP; mmHg & $<90$ or $>200$ & $90-100$ & \\
or $150-160$ & $<90$ or $>160$ \\
Diastoric BP; mmHg & NA & $90-100$ & $>100$ \\
Respiratory rate; & $>25$ & $21-30$ & $<10$ or $>30$ \\
breaths/min & $<92$ & NA & $<95$ \\
Oxygen saturation; \% & NA & $35-37$ & $<35$ or $>38$ \\
Temperature; ${ }^{\circ} \mathrm{C}$ & Altered & Voice & Unresponsive \\
Neurological response & mentation & & Pain \\
Other & Nurse concern & & \\
\hline
\end{tabular}

Additionally, obstetric morbidity (Table 2) of each case was determined by $\mathrm{YH}$.

Table 2. Definition of obstetric morbidity

Severe hypertensive disorder in pregnancy

Eclampsia

Obstetric hemorrhage

Sepsis

Pulmonary edema

Shock

Diabetic ketoacidosis

Intracranial hemorrhage

Asthma

Epilepsy

> The sensitivity and specificity of the KRRS and MEOWS for diagnosing obstetric morbidity were evaluated.

\section{References}

[1] Swanton RD, et al. A national survey of obstetric early warning systems in the United Kingdom. Int J Obstet Anesth 2009;18:253-7.

[2] Singh S, et al. A validation study of the CEMACH recommended modified early obstetric warning system (MEOWS). Anaesthesia 2012; 67: 12-8.

\begin{tabular}{lcc} 
Table 2. Demographic data & & \\
\hline Patient admitted to MFICU & $n$ & 376 \\
Age & yeas & $33.7 \pm 5.3$ \\
Gestational age at admission & weeks & $31(7-39)$ \\
Postpartum & $n(\%)$ & $21(5.6)$ \\
Length of stay & days & $7.5 \pm 5.3$ \\
Obstetric morbidity & $n(\%)$ & $69(18.4)$ \\
\hline
\end{tabular}

Data are presented as mean \pm SD or median (range)

Table 3. Sensitivity and specificity of the KRRS and MEOWS

\begin{tabular}{|c|c|c|c|c|}
\hline & \multicolumn{2}{|c|}{ KRRS } & \multicolumn{2}{|c|}{ MEOWS } \\
\hline & & $95 \% \mathrm{Cl}$ & & $95 \% \mathrm{Cl}$ \\
\hline Sensitivity & 0.47 & $0.42-0.52$ & 0.93 & $0.91-0.96$ \\
\hline Specificity & 0.77 & $0.73-0.81$ & 0.63 & $0.58-0.68$ \\
\hline
\end{tabular}

$\mathrm{Cl}$ : confidence interval

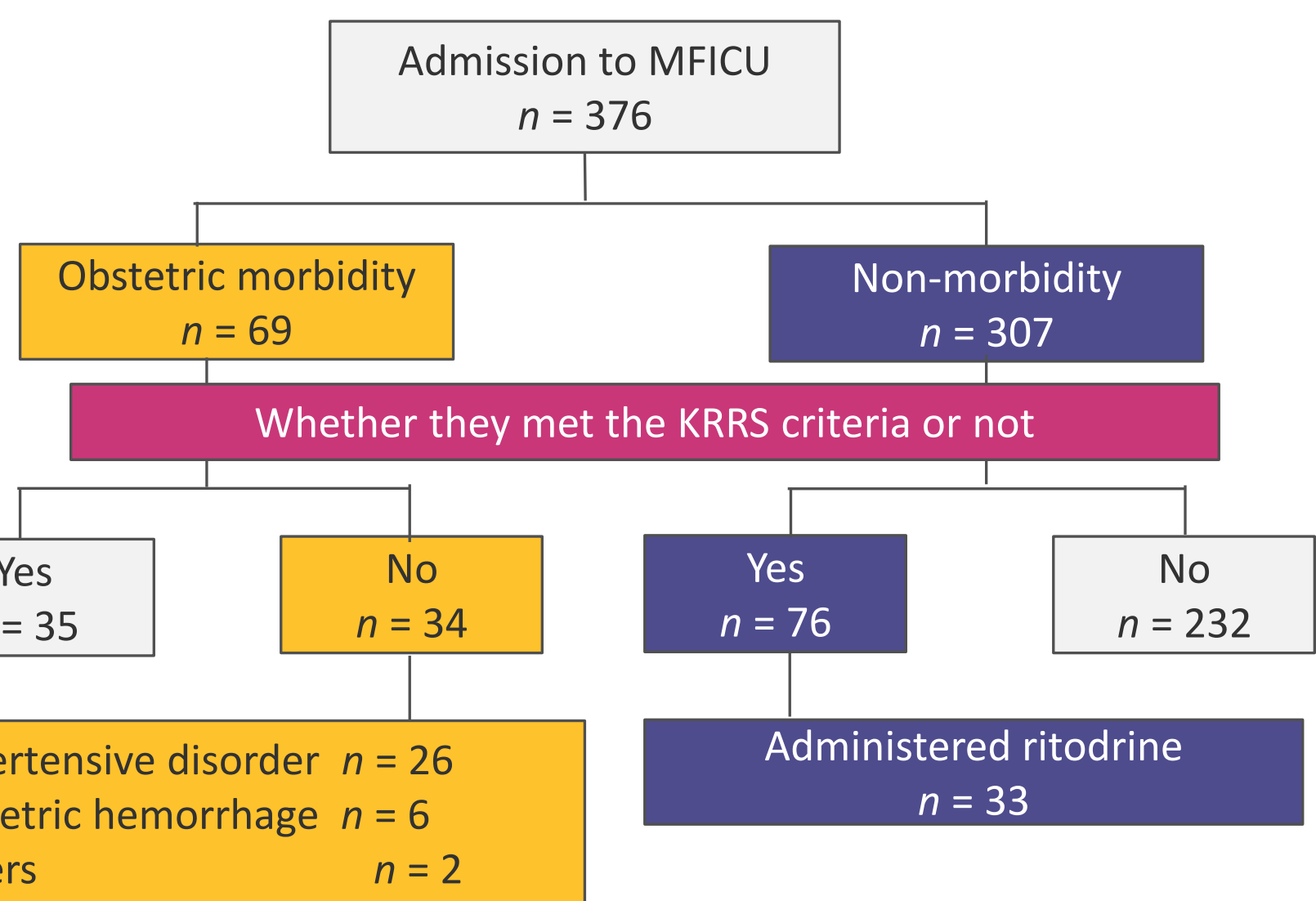

Obstetric hemorrhage $n=6$

Others

$n=2$

Figure1. A flowchart of reviewing cases that meet the KRRS criteria or not

\section{Discussion}

- The sensitivity of the KRRS trigger criteria was $47 \%$.

- This is lower than that of the MEOWS criteria (93\%) in this and a previous study (89\%)[2].

- Lower sensitivity could be attributed to the KRRS trigger criteria not detecting obstetric-specific conditions, including hypertensive disorder in pregnancy and obstetric hemorrhage, which obstetricians frequently encounter.

- The RRS has originally focused on the early prediction of critical conditions that require intensive care, suggesting that the criteria can trigger prompt resources.

- The KRRS was triggered in non-morbidity conditions, particularly with ritodrine, a beta-agonist.

- The KRRS is not adopted to physiological changes in obstetric patients.

- It is imperative to inform RRS members about the specific conditions of obstetric patients.

\section{Conclusion}

- The KRRS criteria can be introduced to the obstetric ward.

- To inform RRS members about the specific conditions of obstetric patients is imperative. 